\title{
Circular Rosenzweig-Porter random matrix ensemble
}

\author{
Wouter Buijsman` and Yevgeny Bar Lev \\ Department of Physics, Ben-Gurion University of the Negev, Beer-Sheva 84105, Israel \\ *buijsman@post.bgu.ac.il
}

November 22, 2021

\begin{abstract}
The Rosenzweig-Porter random matrix ensemble serves as a qualitative phenomenological model for the level statistics and fractality of eigenstates across the many-body localization transition in static systems. We propose a unitary (circular) analogue of this ensemble, which similarly captures the phenomenology of many-body localization in periodically driven (Floquet) systems. We define this ensemble as the outcome of a Dyson Brownian motion process. We show numerical evidence that this ensemble shares some key statistical properties with the Rosenzweig-Porter ensemble for both the eigenvalues and the eigenstates.
\end{abstract}

\section{Contents}

1 Introduction $\quad 1$

2 Rosenzweig-Porter ensemble $\quad 2$

3 Circular analogue $\quad 3$

4 Numerical evaluation $\quad 5$

$\begin{array}{llr}5 & \text { Conclusions and outlook } & 7\end{array}$

$\begin{array}{lr}\text { References } & 8\end{array}$

\section{Introduction}

Many-body localization is known as a robust mechanism of ergodicity breaking in disordered interacting quantum many-body systems that can be thought of as Anderson localization in Fock space $[1,2]$. It is characterized by non-trivial properties, such as the violation of the eigenstate thermalization hypothesis [3] and the persistent memory of an initial state, among many others [4]. Significant progress towards an understanding of many-body localization from both theoretical and experimental approaches has been witnessed during the last one and a half decade [5-7].

Many-body localization can be mapped to single-particle localization on a Fock space lattice [8], an approach which has been successfully utilized both analytically [9-14] and numer- 
ically [15-19]. Within the framework of this approach, obtaining analytical results is notoriously difficult due to strong correlations between disorder potentials [20,21]. It was therefore realized that it is of interest to have simpler phenomenological models for many-body localization that are analytically tractable while still capturing the essential properties [22-24]. A profound example of such a model is the Rosenzweig-Porter random matrix ensemble [25]. This model was suggested as a qualitative phenomenological model for the level statistics and fractality of eigenstates across the many-body localization transition [23], which separates a many-body localized from a thermal phase. This proposal triggered considerable interest for the Rosenzweig-Porter ensemble over the last years [26-35].

The notion of many-body localization extends to interacting periodically driven (Floquet) systems [36-40], for which localization in Fock space has been investigated analytically very recently [41]. While static systems are described by Hamiltonians, which are Hermitian, periodically driven systems are described by Floquet operators, which are unitary. This raises the arguably natural question whether a unitary (or circular since the eigenvalues lie on the unit circle in the complex plane) analogue of the Rosenzweig-Porter ensemble can be constructed.

In this work, we propose a circular analogue of the Rosenzweig-Porter ensemble, which we define as the result of a Dyson Brownian motion process [42]. We provide numerical evidence that this circular analogue has similar features as the Rosenzweig-Porter ensemble by investigating some key statistical properties of the eigenvalues and the eigenstates. Our work might serve as a first step in constructing generalizations covering e.g. multifractality, analog to recent physically motivated proposals for generalizations of the Rosenzweig-Porter ensemble [29,43-46] and other Floquet models with multifractal eigenstates [47-50].

The structure of the paper is as follows. Sec. 2 reviews the Rosenzweig-Porter ensemble and some of its properties. Sec. 3 discusses the construction of the circular Rosenzweig-Porter ensemble. Sec. 4 numerically investigates a number of statistical properties of the circular analogue. Sec. 5 closes the paper with conclusions and an outlook.

\section{Rosenzweig-Porter ensemble}

The Rosenweig-Porter (RP) ensemble [23], which was originally proposed in the context of complex atomic nuclei [25], can be seen as generalization of the Gaussian orthogonal ensemble (GOE) $[51,52]$ with a preferential basis. The ensemble consists of real symmetric matrices $H$ of the form

$$
H=H_{0}+\frac{\epsilon}{\sqrt{N^{\gamma}}} V,
$$

where $N$ is the matrix dimension, $H_{0}$ is diagonal with elements sampled independently from the Gaussian distribution with mean $\mu=0$ and variance $\sigma^{2}=1$, and $V$ is sampled from the GOE. The GOE consists of real-valued symmetric matrices with the diagonal and upper triangular elements sampled independently from the Gaussian distribution with mean $\mu=0$ and variance $\sigma^{2}=2$ (diagonal elements) or $\sigma^{2}=1$ (upper triangular elements). Interpreting $H$ as an Hamiltonian, the parameter $\epsilon \sim \mathcal{O}(1)$ can be viewed of as a perturbation strength. The positive-valued parameter $\gamma$ controls the relative strength of the terms, and thus the properties of the ensemble. Below, we outline a number of these properties, which we numerically explore for the circular analogue in Sec. 4.

Level statistics provide a standard diagnostic for quantum chaos $[3,51]$. In the large- $N$ limit, the eigenvalues of the RP ensemble obey Wigner-Dyson level statistics for $\gamma<2$ [53], which are typically observed for chaotic quantum systems. For $\gamma \geq 2$ and in the same limit, these statistics are Poissonian [53], as typically observed for integrable (non-chaotic) systems. It is convenient to probe level statistics by the average ratio of consecutive level spacings 
[54,55]. Let $E_{n}$ denote the eigenvalues of $H$ sorted in ascending order, such that the spacings $s_{n}$ of consecutive levels are given by $s_{n}=E_{n+1}-E_{n}$. The ratios $r_{n}$ are defined as

$$
r_{n}=\min \left(\frac{s_{n+1}}{s_{n}}, \frac{s_{n}}{s_{n+1}}\right) .
$$

On average, the ratios of consecutive level spacings acquire the values $\bar{r} \approx 0.386$ for Poissonian and $\bar{r} \approx 0.530$ for Wigner-Dyson (GOE) level statistics [55].

Next, we consider eigenstates of $H$ taken from the middle of the spectrum. We focus on the basis in which $H_{0}$ is diagonal. We denote eigenstates of $H$ and $H_{0}$ by respectively $\left|\psi_{n}\right\rangle$ and $|n\rangle$. Localization of an eigenstate $\left|\psi_{n}\right\rangle$ can be quantified by the inverse participation ratios

$$
\operatorname{IPR}_{q}=\sum_{m}\left|\left\langle m \mid \psi_{n}\right\rangle\right|^{2 q}
$$

with $q>1 / 2$. Asymptotically in $N$, the inverse participation ratios scale as $N^{-(q-1) D_{q}}$, where $D_{q}$ is known as the fractal dimension with parameter $q$ [30]. Eigenstates of the RP ensemble are characterized by $D_{q}=1$ for $\gamma \leq 1$, indicating that these are spread out over a finite fraction of the Hilbert space as IPR $2 \sim N^{-1}$. For $1<\gamma<2$, the eigenstates are fractal with $D_{q}=2-\gamma$. As $D_{q}$ does not depend on $q$, the eigenstates are fractal but not multifractal. For $\gamma \geq 2$, the eigenstates are characterized by $D_{q}=0$, which reflects that these eigenstates have significant overlap with only $\mathcal{O}(1)$ basis states [23].

Eigenstates of the RP ensemble obey Breit-Wigner statistics [30], which are expected to apply rather generically to quantum many-body systems [56-59]. In the large- $N$ limit, it follows that

$$
\overline{\left|\left\langle m \mid \psi_{n}\right\rangle\right|^{2}} \sim \frac{1}{\left(E_{n}-E_{m}^{(0)}\right)^{2}+\Gamma^{2}\left(E_{n}\right)},
$$

where the bar denotes an average over $V$ in Eq. (1), $E_{n}$ is the eigenvalue corresponding to the eigenstate $\left|\psi_{n}\right\rangle, E_{n}^{(0)}=\left(H_{0}\right)_{n n}$, and the so-called spreading width $\Gamma\left(E_{n}\right)$ is obtained through Fermi's golden rule as

$$
\Gamma\left(E_{n}\right)=\frac{\pi \epsilon^{2}}{N^{\gamma}} \rho\left(E_{n}\right),
$$

where $\rho\left(E_{n}\right)=\sum_{i} \delta\left(E_{n}-E_{i}\right)$ is the density of states in the vicinity of the eigenvalue $E_{n}$. Eq. (4) describes what is known as the shape of the eigenstates [59]. For $1<\gamma<2$, the spreading width gives the width of the so-called mini-band $[23,31,60]$. In this energy window, the eigenstate amplitudes $\left|\left\langle\psi_{n} \mid m\right\rangle\right|^{2}$ are on average of order $N^{-D_{2}}$ (where $D_{2}$ is the fractal dimension $D_{q}$ for $q=2$ ), which is much larger than the value $N^{-1}$ obtained when averaging over all basis states.

\section{Circular analogue}

In this Section, we propose a unitary analogue of the RP ensemble, which we refer to as the circular RP (CRP) ensemble. The starting point of our construction is the observation made in Ref. [26] that the RP ensemble can be seen as the outcome of a finite-time stochastic process for the matrix elements, referred to as a Dyson Brownian motion process [42]. Let $M(t)$ denote a time-dependent, real symmetric matrix of dimension $N$, which evolves stochastically according to

$$
d M(t)=\sqrt{d t} X
$$

where $d M(t)=M(t+d t)-M(t)$ and $X$ is a GOE matrix sampled independently at each infinitesimal time step $d t$. For the initial condition $M(0)=H_{0}$ with $H_{0}$ as given in Eq. (1), 
one finds $M(t)=H_{0}+\sqrt{t} V$, where $V$ is a sample from the GOE. From the solution $M(t)$ is it clear that the RP ensemble results at $t=\epsilon^{2} / N^{\gamma}$. The GOE is invariant under transformations of the basis $[51,52]$. Using this, one can obtain stochastic equations for the eigenvalues $E_{n}(t)$ of $M(t)$ perturbatively as

$$
d E_{n}(t)=\sqrt{d t} X_{n n}+d t \sum_{m \neq n} \frac{\left(X_{n m}\right)^{2}}{E_{n}(t)-E_{m}(t)},
$$

and the stochastic equations for the corresponding eigenstates $\left|\psi_{n}\right\rangle$ as

$$
d\left|\psi_{n}(t)\right\rangle=\sqrt{d t} \sum_{m \neq n} \frac{X_{m n}}{E_{n}(t)-E_{m}(t)}\left|\psi_{m}(t)\right\rangle-\frac{d t}{2} \sum_{m \neq n} \frac{\left(X_{m n}\right)^{2}}{\left[E_{n}(t)-E_{m}(t)\right]^{2}}\left|\psi_{n}(t)\right\rangle,
$$

where the increments are given by $d E_{n}(t)=E_{n}(t+d t)-E_{n}(t)$ and $\left.d\left|\psi_{n}(t)\right\rangle=\left|\psi_{n}(t+d t)\right\rangle-\psi_{n}(t)\right\rangle$. Note that these equations are exact in the limit $d t \rightarrow 0$. In the literature, the stochastic evolution of the eigenstates is known as the eigenvector moment flow [61,62].

To construct a circular ensemble, we follow an approach introduced by Dyson [42]. We introduce $S(t)$, which is a time-dependent symmetric unitary matrix of dimension $N$ with eigenvalues $e^{i \theta_{n}(t)}$. Such a matrix can be interpreted as the Floquet operator of a periodically driven system obeying time-reversal symmetry. The matrix evolves stochastically according to

$$
d S(t)=i \sqrt{d t} U^{T}(t) X U(t),
$$

where $d S(t)=S(t+d t)-S(t)$, and $U(t)$ is a unitary matrix defined via the decomposition $S(t)=U^{T}(t) U(t)$. The decomposition is defined up to transformations $U \rightarrow O U$ for real orthogonal matrices $O^{1}$, which can be absorbed in $X$ as the GOE is invariant under basis transformations. We propose the CRP ensemble as the outcome of the stochastic process described by Eq. (9) at $t=\epsilon^{2} / N^{\gamma}$. For the RP ensemble, $M(0)$ is diagonal with uncorrelated elements. This motivates us to analogously take

$$
S(0)=\operatorname{diag}\left(e^{i \theta_{1}(0)}, \ldots, e^{i \theta_{N}(0)}\right)
$$

with the phases $\theta_{n}(0)$ sampled independently from the uniform distribution ranging over $[-\pi, \pi)$. This non-unique choice respects the uniform density of states as observed generically for Floquet operators. The phases evolve according to a stochastic equation that can be obtained as

$$
d \theta_{n}(t)=\sqrt{d t} X_{n n}+d t \sum_{m \neq n} \frac{\left(X_{n m}\right)^{2}}{2 \tan \frac{1}{2}\left[\theta_{n}(t)-\theta_{m}(t)\right]},
$$

where $d \theta_{n}(t)=\theta_{n}(t+d t)-\theta_{n}(t)$.

Eqs. (7) and (11) are known to yield the same statistics in the middle of the spectrum and in the large- $N$ limit, provided that the densities of states of $M(t)$ and $S(t)$ are the same [63]. Heuristically, this can be understood by noticing that the summations are dominated by contributions from the few most nearby levels, which are of the order of the density of states, $\rho\left(E_{n}\right) \sim N$. While there are $\mathcal{O}(N)$ additional terms in these summations, at the middle of the spectrum the contributions from levels $E_{m}>E_{n}\left(\theta_{m}>\theta_{n}\right)$ and $E_{m}<E_{n}\left(\theta_{m}<\theta_{n}\right)$ are respectively negative and positive, and therefore provide a contribution with an overall sub-leading magnitude of $\mathcal{O}(\sqrt{N})$. The density of states of $S(t)$ can be made equal to those of $M(t)$ by scaling the latter by $1 / \sqrt{2 \pi}$. We therefore expect the spectral statistics of the RP

\footnotetext{
${ }^{1}$ The set $U_{S}(N)$ of symmetric unitary matrices of dimension $N$ is isomorphic to $U(N) / O(N)$, where $U(N)$ denotes the set of imaginary unitary matrices of dimension $N$, and $O(N)$ denote the set of real orthogonal matrices of dimension N. For a proof, see e.g. Proposition 2.2.4 of Ref. [52].
} 
for a given $\epsilon$ at the middle of the spectrum to match those of the CRP with $\epsilon \rightarrow \epsilon / \sqrt{2 \pi}$. We remark that $M(t)$ has a density of states that remains constant during the Brownian motion process for $\gamma>1$ [30].

The eigenstates $\left|\psi_{n}\right\rangle$ of $S(t)$ associated with the eigenvalues $e^{i \theta_{n}(t)}$ evolve stochastically according to

$d\left|\psi_{n}(t)\right\rangle=\sqrt{d t} \sum_{m \neq n} \frac{X_{m n}}{2 \tan \frac{1}{2}\left[\theta_{n}(t)-\theta_{m}(t)\right]}\left|\psi_{m}(t)\right\rangle-\frac{d t}{2} \sum_{m \neq n} \frac{(X)_{m n}^{2}}{4 \tan ^{2} \frac{1}{2}\left[\theta_{n}(t)-\theta_{m}(t)\right]}\left|\psi_{n}(t)\right\rangle$,

where again $d\left|\psi_{n}(t)\right\rangle=\left|\psi_{n}(t+d t)\right\rangle-\left|\psi_{n}(t)\right\rangle$. In line with the arguments for the eigenvalues, one might expect that the RP and CRP have similar eigenstate statistics. We remark that no explicit construction for the matrices resulting from circular Dyson Brownian motion processes is known [63]. Moreover, we note that the CRP can not be constructed as $S=e^{i H}$ for $H$ sampled from the RP ensemble. Indeed, for example then the density of states of $S$ would not be uniform.

\section{Numerical evaluation}

The results below are obtained through a numerical evaluation of Eq. (9) up to $t=\epsilon^{2} / N^{\gamma}$ using the algorithm described Ref. [52] (Sec. 11.2.1). We average over at least $10^{6}$ eigenvalues or eigenstates. In the results below, we take $\epsilon=1$ and $10^{4}$ time steps to reach $t=\epsilon^{2} / N^{\gamma}$. We have validated that our results are visibly indistinguishable from results obtained when taking the number of time steps half as large, and found that no qualitative differences can be observed when taking the number of time steps an order of magnitude smaller. The numerical evaluation of Eq. (9) requires diagonalization or matrix-matrix multiplications in each step, making it is computationally expensive. We therefore restricted to matrix dimensions $N \leq 1000$, which still provide sufficient numerical evidence for the claims of this work.

We denote the eigenphases for samples from the CRP ensemble by $\theta_{n}$. First, we consider the average ratio of consecutive level spacings $\bar{r}$. The ratios $r_{n}$ of consecutive level spacings are defined in Eq. (2), with $E_{n}$ replaced by the phases $\theta_{n}$. The left panel of Fig. 1 shows $\bar{r}$ as a function of $\gamma$ for several matrix dimensions. As discussed in Sec. 2, the RP ensemble is characterized by $\bar{r} \approx 0.530$ for $\gamma<2$ (Wigner-Dyson) and $\bar{r} \approx 0.386$ (Poissonian) for $\gamma \geq 2$ in the large- $N$ limit, which is consistent with our results. The level statistics exhibit a first-order $(v=1)$ phase transition from Wigner-Dyson to Poissonian at $\gamma=\gamma_{c}=2$. For the RP ensemble, a scaling collapse of $\bar{r}$ as a function of $\left(\gamma-\gamma_{c}\right) \ln (N)^{1 / v}$ has been observed in Ref. [33]. The right panel shows that this observation can be made as well for the CRP ensemble.

Next, we consider eigenstates of the CRP ensemble. We focus on the basis in which $S(0)$ is diagonal. We denote eigenstates of $S$ and $S_{0}$ by respectively $\left|\psi_{n}\right\rangle$ and $|n\rangle$, with the eigenvalue $e^{i \theta_{n}}$ corresponding to $\left|\psi_{n}\right\rangle$. We first focus on the eigenstate inverse participation ratio $\mathrm{IPR}_{2}$ as given in Eq. (3). Asymptotically in $N$, the RP ensemble is characterized by $\mathrm{IPR}_{2} \sim N^{-1}$ for $\gamma \leq 1$, and $\mathrm{IPR}_{2}=\mathcal{O}(1)$ for $\gamma \geq 2$ as discussed in Sec. 2. In the intermediate region $1<\gamma<2$, the eigenstates are fractal, and are characterized by $\mathrm{IPR}_{2} \sim N^{-(2-\gamma)}$. Fig. 2 shows the average $\overline{\mathrm{IPR}}_{2}$ (top panel) and $N \times \overline{\mathrm{IPR}}_{2}$ (bottom panel) as a function of $\gamma$, for the same matrix dimensions as studied above. The results we obtain for the CRP ensemble are again consistent with the large- $N$ behavior for the RP ensemble.

Finally, we consider the shape of the eigenstates. The eigenstates of the RP ensemble obey Breit-Wigner statistics [30]. For the CRP ensemble we aim to show [cf. Eq. (4)] that

$$
\overline{\left|\left\langle m \mid \psi_{n}\right\rangle\right|^{2}} \sim \frac{1}{\left(\theta_{n}-\theta_{m}^{(0)}\right)^{2}+\Gamma^{2}},
$$



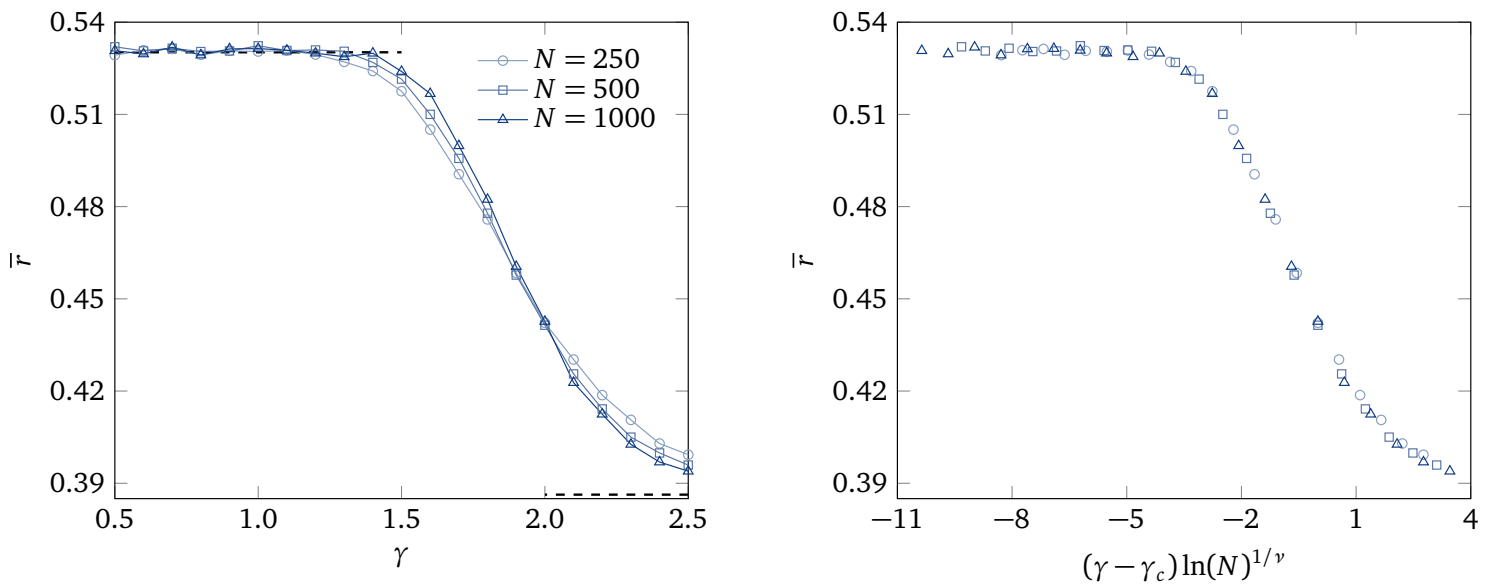

Figure 1: The average ratio of consecutive level spacing spacings $\bar{r}$ as a function of $\gamma$ (left panel) and $\left(\gamma-\gamma_{c}\right) \ln (N)^{1 / v}$ with $\gamma_{c}=2$ and $v=1$ (right panel) for matrix dimensions $N=250,500$, and 1000. Poissonian and Wigner-Dyson level statistics are characterized by respectively $\bar{r} \approx 0.386$ and $\bar{r} \approx 0.530$ (dashed lines).
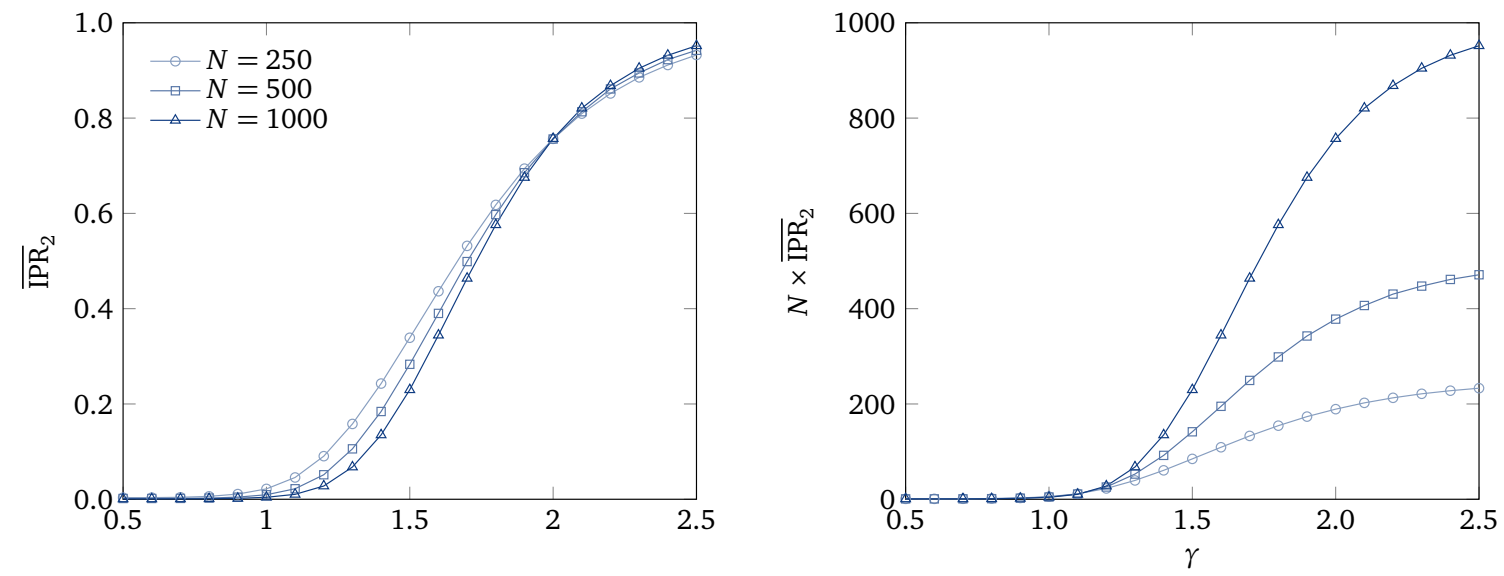

Figure 2: The average of the inverse participation ratio $\mathrm{IPR}_{2}$ (left panel) and the value scaled by $N$ (right panel) as a function of $\gamma$ for matrix dimensions $N=250$, 500, and 1000 . 

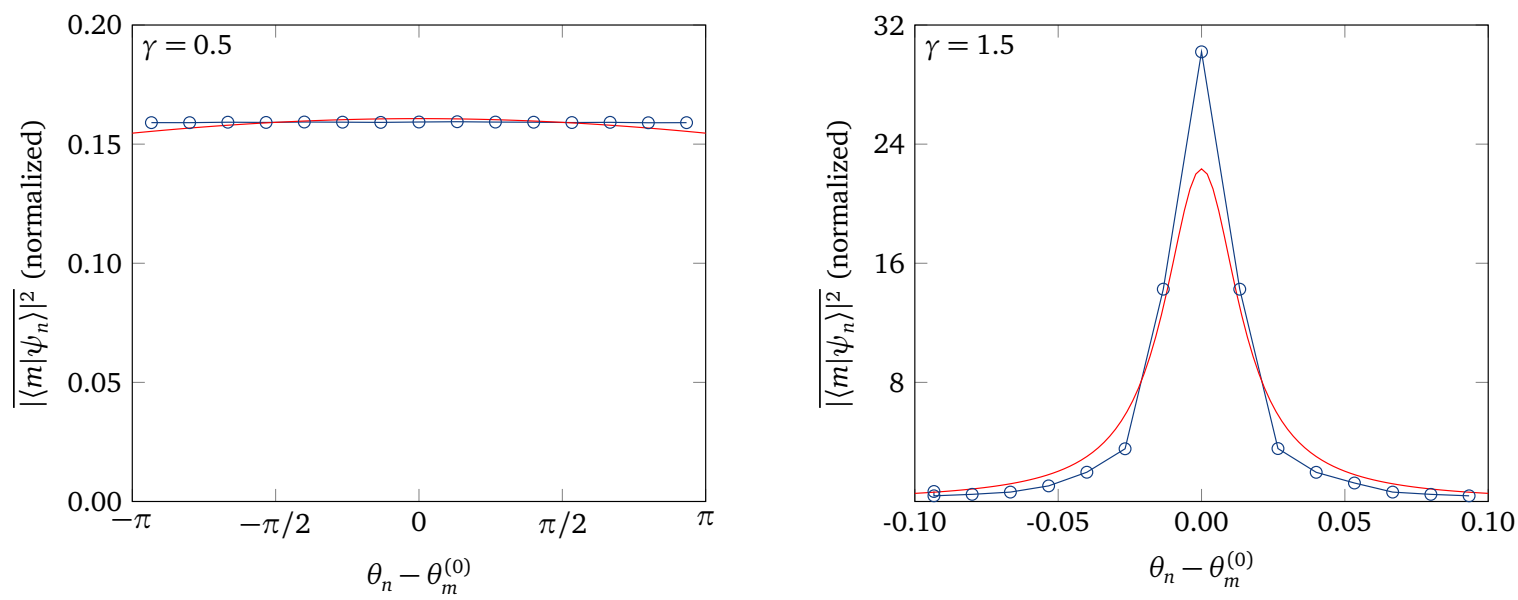

Figure 3: The average of $\left|\left\langle m \mid \psi_{n}\right\rangle\right|^{2}$ as a function of $\theta_{n}-\theta_{m}^{(0)}$ (markers), combined with the evaluation of Eq. (13) with $\rho=1000 /(2 \pi)$ (red solid lines) for $N=1000$ with $\gamma=0.5$ (left panel) and $\gamma=1.5$ (right panel). Each of the curves is normalized to unit area over the plotted range. Blue lines connecting the markers serve as a guide to the eye.

where $\theta_{m}^{(0)}=\theta_{m}(0)$ as introduced in Eq. (10). Fig. 3 shows the average $\overline{\left|\left\langle m \mid \psi_{n}\right\rangle\right|^{2}}$ as a function of $\theta_{n}-\theta_{m}^{(0)}$ for $\gamma=0.5$ (top panel) and $\gamma=1.5$ (bottom panel) for the largest matrix dimension $N=1000$. The smooth curves show the evaluation of Eq. (13) with $\Gamma$ obtained from Eq. (5), and the density of states is given by $\rho=1000 /(2 \pi) \approx 159.2$.

For $\gamma=0.5$ the expected spreading width has a value $\Gamma \approx 15.8$, such that the resulting Lorentzian shape has a width exceeding the width $2 \pi$ of the spectrum, leading to a slight mismatch with the observed results. For $\gamma=1.5$ the spreading width evaluates to $\Gamma \approx 1.6 \times 10^{-3}$, which is comparable to the mean level spacing $\rho^{-1} \approx 6.3 \times 10^{-4}$. Eq. (4) relies on a continuum approximation for the density of states [30]. Presumably due to this, the agreement between our data and the expected results is not perfect, although qualitative agreement, and a pronounced difference between the structures of the eigenstates for $\gamma=0.5$ and $\gamma=1.5$ is clearly seen. To our knowledge, this characteristic of eigenstates has not been previously investigated for unitary matrix ensembles.

\section{Conclusions and outlook}

We have proposed a unitary (circular) analogue of the Rosenzweig-Porter (RP) ensemble, defined as the outcome of a Dyson Brownian motion process. We have numerically verified that some key statistics of both the eigenvalues and the eigenstates of the circular analogue match the behaviour of the RP ensemble. The circular analogue, therefore, can serve as a phenomenological model for the level statistics and fractality of eigenstates as observed across the many-body localization transition for periodically driven systems.

Motivated by, among other observations, the suggestion that eigenstates near the manybody localization transition are multifractal [64-66], several generalizations of the RP ensemble have been proposed [29,43-46]. It would be interesting to see if similar generalization could be constructed for the circular RP ensemble, next to other Floquet models with multifractal eigenstates [47-50]. This might for example be achievable by considering stochastic processes with correlated increments, which has been initialized for the RP ensemble recently [67]. Second, the circular RP ensemble could potentially be of value in studies on ran- 
dom quantum circuits as a non-maximally random building block, analog to e.g. the proposal made in Ref. [68].

\section{Acknowledgements}

We thank Ivan M. Khaymovich for the careful reading of the manuscript and providing us with many useful comments, as well as Lea F. Santos for useful discussions.

Funding information YB acknowledges support from the Israel Science Foundation (grants No. 218/19 and 527/19). WB acknowledges support from the Kreitman School of Advanced Graduate Studies at Ben-Gurion University.

\section{References}

[1] D. M. Basko, I. L. Aleiner and B. L. Altshuler, Metal-insulator transition in a weakly interacting many-electron system with localized single-particle states, Ann. Phys. 321, 1126 (2006), doi:10.1016/j.aop.2005.11.014.

[2] I. V. Gornyi, A. D. Mirlin and D. G. Polyakov, Interacting Electrons in Disordered Wires: Anderson Localization and Low-T Transport, Phys. Rev. Lett. 95, 206603 (2005), doi:10.1103/PhysRevLett.95.206603.

[3] L. D'Alessio, Y. Kafri, A. Polkovnikov and M. Rigol, From quantum chaos and eigenstate thermalization to statistical mechanics and thermodynamics, Adv. Phys. 65, 239 (2016), doi:10.1080/00018732.2016.1198134.

[4] D. Huse, R. Nandkishore and V. Oganesyan, Phenomenology of fully many-body-localized systems, Phys. Rev. B 90, 174202 (2014), doi:10.1103/PhysRevB.90.174202.

[5] R. Nandkishore and D. A. Huse, Many-Body Localization and Thermalization in Quantum Statistical Mechanics, Annu. Rev. Condens. Matter Phys. 6, 15 (2015), doi:10.1146/annurev-conmatphys-031214-014726.

[6] F. Alet and N. Laflorencie, Many-body localization: An introduction and selected topics, C.R. Phys. 19, 498 (2018), doi:10.1016/j.crhy.2018.03.003.

[7] D. A. Abanin, E. Altman, I. Bloch and M. Serbyn, Colloquium: Many-body localization, thermalization, and entanglement, Rev. Mod. Phys. 91, 021001 (2019), doi:10.1103/RevModPhys.91.021001.

[8] B. L. Altshuler, Y. Gefen, A. Kamenev and L. S. Levitov, Quasiparticle Lifetime in a Finite System: A Nonperturbative Approach, Phys. Rev. Lett. 78, 2803 (1997), doi:10.1103/PhysRevLett.78.2803.

[9] C. Monthus and T. Garel, Many-body localization transition in a lattice model of interacting fermions: Statistics of renormalized hoppings in configuration space, Phys. Rev. B 81, 134202 (2010), doi:10.1103/PhysRevB.81.134202.

[10] M. Serbyn, Z. Papić and D. A. Abanin, Local Conservation Laws and the Structure of the Many-Body Localized States, Phys. Rev. Lett. 111, 127201 (2013), doi:10.1103/PhysRevLett.111.127201. 
[11] V. Ros, M. Müller and A. Scardicchio, Integrals of motion in the many-body localized phase, Nucl. Phys. B 891, 420 (2015), doi:10.1016/j.nuclphysb.2014.12.014.

[12] F. Pietracaprina, V. Ros and A. Scardicchio, Forward approximation as a mean-field approximation for the Anderson and many-body localization transitions, Phys. Rev. B 93, 054201 (2016), doi:10.1103/PhysRevB.93.054201.

[13] S. Roy and D. E. Logan, Strong ergodicity breaking due to local constraints in a quantum system, SciPost Phys. 7, 042 (2019), doi:10.21468/SciPostPhys.7.4.042.

[14] S. Roy and A. Lazarides, Strong ergodicity breaking due to local constraints in a quantum system, Phys. Rev. Research 2, 023159 (2020), doi:10.1103/PhysRevResearch.2.023159.

[15] S. Bera, H. Schomerus, F. Heidrich-Meisner and J. H. Bardarson, Many-Body Localization Characterized from a One-Particle Perspective, Phys. Rev. Lett. 115, 046603 (2015), doi:10.1103/PhysRevLett.115.046603.

[16] B. Villalonga, X. Yu, D. J. Luitz and B. K. Clark, Exploring one-particle orbitals in large many-body localized systems, Phys. Rev. B 97, 104406 (2018), doi:10.1103/PhysRevB.97.104406.

[17] W. Buijsman, V. Gritsev and V. Cheianov, Many-body localization in the Fock space of natural orbitals, Scipost Phys. 4, 038 (2018), doi:10.21468/SciPostPhys.4.6.038.

[18] M. Hopjan and F. Heidrich-Meisner, Many-body localization from a one-particle perspective in the disordered one-dimensional Bose-Hubbard model, Phys. Rev. A 101, 063617 (2020), doi:10.1103/PhysRevA.101.063617.

[19] S. Roy and D. E. Logan, Fock-space anatomy of eigenstates across the many-body localization transition, Phys. Rev. B 104, 174201 (2021), doi:10.1103/PhysRevB.104.174201.

[20] A. Altland and T. Micklitz, Field Theory Approach to Many-Body Localization, Phys. Rev. Lett. p. 127202 (2017), doi:10.1103/PhysRevLett.118.127202.

[21] S. Roy and D. E. Logan, Fock-space correlations and the origins of many-body localization, Phys. Rev. B 101, 134202 (2020), doi:10.1103/PhysRevB.101.134202.

[22] A. De Luca, B. L. Altshuler, V. E. Kravtsov and A. Scardicchio, Anderson Localization on the Bethe Lattice: Nonergodicity of Extended States, Phys. Rev. Lett. 113, 046806 (2014), doi:10.1103/PhysRevLett.113.046806.

[23] V. E. Kravtsov, I. M. Khaymovich, E. Cuevas and M. Amini, A random matrix model with localization and ergodic transitions, New J. Phys. 17, 122002 (2015), doi:10.1088/13672630/17/12/122002.

[24] K. S. Tikhonov and A. D. Mirlin, From Anderson localization on random regular graphs to many-body localization (2021), 2102.05930.

[25] N. Rosenzweig and C. E. Porter, "Repulsion of Energy Levels" in Complex Atomic Spectra, Phys. Rev. 120, 1698 (1960), doi:10.1103/PhysRev.120.1698.

[26] D. Facoetti, P. Vivo and G. Biroli, From non-ergodic eigenvectors to local resolvent statistics and back: A random matrix perspective, EuroPhys. Lett. 115, 47003 (2016), doi:10.1209/0295-5075/115/47003. 
[27] K. Truong and A. Ossipov, Eigenvectors under a generic perturbation: Non-perturbative results from the random matrix approach, New J. Phys. 116, 37002 (2016), doi:10.1209/0295-5075/116/37002.

[28] M. Amini, Spread of wave packets in disordered hierarchical lattices, Europhys. Lett. 117, 30003 (2017), doi:10.1209/0295-5075/117/30003.

[29] C. Monthus, Multifractality of eigenstates in the delocalized non-ergodic phase of some random matrix models: Wigner-Weisskopf approach, J. Phys. A: Math. Theor. 50, 295101 (2017), doi:10.1088/1751-8121/aa77e1.

[30] E. Bogomolny and M. Sieber, Eigenfunction distribution for the Rosenzweig-Porter model, Phys. Rev. E 98, 032139 (2018), doi:10.1103/PhysRevE.98.032139.

[31] G. De Tomasi, M. Amini, S. Bera, I. M. Khaymovich and V. E. Kravtsov, Survival probability in Generalized Rosenzweig-Porter random matrix ensemble, SciPost Phys. 6, 014 (2019), doi:10.21468/SciPostPhys.6.1.014.

[32] P. von Soosten and S. Warzel, Non-ergodic delocalization in the Rosenzweig-Porter model, Lett. Math. Phys. 109, 905 (2019), doi:10.1007/s11005-018-1131-7.

[33] M. Pino, J. Tabanera and P. Serna, From ergodic to non-ergodic chaos in Rosenzweig-Porter model, J. Phys. A: Math. Theor. 52, 475101 (2019), doi:10.1088/1751-8121/ab4b76.

[34] R. Berkovits, Super-Poissonian behavior of the Rosenzweig-Porter model in the nonergodic extended regime, Phys. Rev. B 102, 165140 (2020), doi:10.1103/PhysRevB.102.165140.

[35] M. Tarzia, Many-body localization transition in Hilbert space, Phys. Rev. B 102, 014208 (2020), doi:10.1103/PhysRevB.102.014208.

[36] P. Ponte, Z. Papić, F. Huveneers and D. A. Abanin, Many-Body Localization in Periodically Driven Systems, Phys. Rev. Lett. 114, 140401 (2015), doi:10.1103/PhysRevLett.114.140401.

[37] A. Lazarides, A. Das and R. Moessner, Fate of Many-Body Localization Under Periodic Driving, Phys. Rev. Lett. 115, 030402 (2015), doi:10.1103/PhysRevLett.115.030402.

[38] D. A. Abanin, W. de Roeck and F. Huveners, Theory of many-body localization in periodically driven systems, Ann. Phys. 372, 1 (2016), doi:10.1016/j.aop.2016.03.010.

[39] L. Zhang, V. Khemani and D. A. Huse, A Floquet model for the many-body localization transition, Phys. Rev. B 94, 224202 (2016), doi:10.1103/PhysRevB.94.224202.

[40] P. Bordia, H. Lüschen, U. Schneider, M. Knap and I. Bloch, Periodically driving a manybody localized quantum system, Nat. Phys. 13, 460 (2017), doi:10.1038/nphys4020.

[41] S. Roy, R. Moessner and A. Lazarides, How periodic driving stabilizes and destabilizes Anderson localization on random trees, Phys. Rev. B 103, L100204 (2021), doi:10.1103/PhysRevB.103.L100204.

[42] F. J. Dyson, A Brownian-Motion Model for the Eigenvalues of a Random Matrix, J. Math. Phys. 3, 1196 (1962), doi:10.1063/1.1703862.

[43] V. E. Kravtsov, I. M. Khaymovich, B. L. Altshuler and L. B. Ioff, Localization transition on the Random Regular Graph as an unstable tricritical point in a log-normal RosenzweigPorter random matrix ensemble (2020), 2002.02979. 
[44] I. M. Khaymovich, V. E. Kravtsov, B. L. Altshuler and L. B. Ioffe, Fragile extended phases in the log-normal Rosenzweig-Porter model, Phys. Rev. Research 2, 043346 (2020), doi:10.1103/PhysRevResearch.2.043346.

[45] G. Biroli and M. Tarzia, Lévy-Rosenzweig-Porter random matrix ensemble, Phys. Rev. B 103, 104205 (2021), doi:10.1103/PhysRevB.103.104205.

[46] I. M. Khaymovich and V. E. Kratsov, Dynamical phases in a "multifractal" Rosenzweig-Porter model, SciPost Phys. 11, 45 (2021), doi:10.21468/SciPostPhys.11.2.045.

[47] J. N. Bandyopadhyay, J. Wang and J. Gong, Generating a fractal butterfly Floquet spectrum in a class of driven SU(2) systems: Eigenstate statistics, Phys. Rev. E 81, 066212 (2010), doi:10.1103/PhysRevE.81.066212.

[48] S. Ray, A. Ghosh and S. Sinha, Drive-induced delocalization in the Aubry-André model, Phys. Rev. E 97, 010101 (2018), doi:10.1103/PhysRevE.97.010101.

[49] S. Roy, I. M. Khaymovich, A. Das and R. Moessner, Multifractality without fine-tuning in a Floquet quasiperiodic chain, SciPost Phys. 4, 025 (2018), doi:10.21468/SciPostPhys.4.5.025.

[50] M. Sarkar, R. Ghosh, A. Sen and K. Sengupta, Mobility edge and multifractality in a periodically driven Aubry-André model, Phys. Rev. B 103, 184309 (2021), doi:10.1103/PhysRevB.103.184309.

[51] M. L. Mehta, Random Matrices, vol. 142 of Pure and Applied Mathematics, Elsvier, New York, 3 edn. (2004).

[52] P. J. Forrester, Log-Gases and Random Matrices, vol. 34 of London Mathematical Society Monographs, Princeton University Press, Princeton and Oxford (2010).

[53] A. Altland, M. Janssen and B. Shapiro, Perturbation theory for the Rosenzweig-Porter matrix model, Phys. Rev. E 56, 1471 (1997), doi:10.1103/PhysRevE.56.1471.

[54] V. Oganesyan and D. A. Huse, Localization of interacting fermions at high temperature, Phys. Rev. B 75, 155111 (2007), doi:10.1103/PhysRevB.75.155111.

[55] Y. Y. Atas, E. Bogomolny, O. Giraud and G. Roux, Distribution of the Ratio of Consecutive Level Spacings in Random Matrix Ensembles, Phys. Rev. Lett. 110, 084101 (2013), doi:10.1103/PhysRevLett.110.084101.

[56] E. P. Wigner, Characteristic Vectors of Bordered Matrices With Infinite Dimensions, Ann. Math. 62, 548 (1955), doi:10.2307/1970079.

[57] P. Jacquod and D. L. Shepelyansky, Hidden Breit-Wigner Distribution and Other Properties of Random Matrices with Preferential Basis, Phys. Rev. Lett. 75, 3501 (1995), doi:10.1103/PhysRevLett.75.3501.

[58] Y. V. Fyodorov, O. A. Chubykalo, F. M. Izrailev and G. Casati, Wigner Random Banded Matrices with Sparse Structure: Local Spectral Density of States, Phys. Rev. Lett. 76, 1603 (1996), doi:10.1103/PhysRevLett.76.1603.

[59] F. Borgonovi, F. M. Izrailev, L. F. Santos and V. G. Zelevinsky, Quantum chaos and thermalization in isolated systems of interacting particles, Phys. Rep. 626, 1 (2016), doi:10.1016/j.physrep.2016.02.005. 
[60] E. Cuevas and V. E. Kravtsov, Two-eigenfunction correlation in a multifractal metal and insulator, Phys. Rev. B 76, 235119 (2007), doi:10.1103/PhysRevB.76.235119.

[61] R. Allez, J. Bun and J.-P. Bouchaud, The eigenvectors of Gaussian matrices with an external source (2015), 1412.7108v4.

[62] P. Bourgade and H.-T. Yau, The eigenvector moment flow and local quantum unique ergodicity, Commun. Math. Phys. 350, 231 (2017), doi:10.1007/s00220-016-2627-6.

[63] P. J. Forrester and T. Nagao, Correlations for the circular Dyson Brownian motion model with Poisson initial conditions, Nucl. Phys. B 532, 733 (1998), doi:10.1016/S05503213(98)00551-3.

[64] M. Serbyn, Z. Papić and D. A. Abanin, Thouless energy and multifractality across the many-body localization transition, Phys. Rev. B 96, 104201 (2017), doi:10.1103/PhysRevB.96.104201.

[65] N. Macé, F. Alet and N. Laflorencie, Multifractal Scalings Across the Many-Body Localization Transition, Phys. Rev. Lett. 123, 180601 (2019), doi:10.1103/PhysRevLett.123.180601.

[66] D. J. Luitz, I. M. Khaymovich and Y. Bar Lev, Multifractality and its role in anomalous transport in the disordered XXZ spin-chain, SciPost Phys. Core 2, 006 (2020), doi:10.21468/SciPostPhysCore.2.2.006.

[67] A. G. Kutlin and I. M. Khaymovich, Emergent fractal phase in energy stratified random models (2021), 2106.03864v2.

[68] A. Chan, A. De Luca and J. T. Chalker, Spectral Statistics in Spatially Extended Chaotic Quantum Many-Body Systems, Phys. Rev. Lett. 121, 060601 (2018), doi:10.1103/PhysRevLett.121.060601. 